\title{
The Research of Spread Spectrum Communication Based on the Realization of FPGA
}

\author{
ZHANG Da-Jiang, WANG Jian-Ye, LIU Jun-Jie \\ Air and Missile Defense College, Air Force Engineering University, Xi'an, 710051, China
}

Keywords: spread spectrum communication; pseudo random sequence code; anti-jamming; the function of encryption

\begin{abstract}
It gives the functional block diagram of the transmitter of the spread spectrum communication (SSC) with the spread spectrum code of Pseudo-Noise code and the modulated way of ASK (amplitude-shift keying), and then realized the common ASK transmitter and the corresponding SSC method on the FPGA (field programmable gate array). Then we have a comparison between the wave outputted from the two transmitters to analysis the ability of anti-jamming and the function of encryption of the SSC, and finally we get the superiority of the SSC.
\end{abstract}

\section{Introduction}

The ultra-wideband communication (UWBC) has got the extensive attention now, and their transmission of low-energy density has provided some basis for the low interpret and capture ratio and the high anti-jamming ratio, and the SSC is the one of the UWBC. The SSC is spread spectrum communicated, it combines with the fiber-optical communication, satellite communication are praised to be the three high-technology communication after the message times. And the SSC is the important development direction of the contemporary communication technique, and it has the advantages of the high anti-jamming, the low intercept and capture, code division multiple address, signal hidden and encryption because of the using of pseudo random code. Formally, the SSC was used in the military communication, the electrical rival, the navigation and the measure, and now, it has an extensive using in the civil communication territory.

Because FPGA has the advantages in the property, cost, flexibility and power dissipation, the signal processing based on the FPGA has applied in the various signal processing territory extensively. The design of the communication system based on FPGA which has a flexible application on the every model can satisfy the various need of the standard and the increasing changeable need of the communication in the function. And in the article, I use the design of FPGA to satisfy the choosing while we need have a comparison of the function.

In the research of the related territory, the reference [4] promotes a restrained method to the time domain cancellation of the frequency domain interference, and it deduces the theoretical formula of the bit error ratio, which provides some theoretical basis of the analysis of the anti-jamming of the SSC; the reference [5] has some researches of the new spread spectrum sequence, it provide some theoretical basis of the enrichment of the spread spectrum code and the extension of the function. The article based on the theory of the reference [4] and [5], and used the FPGA as the test platform, realizing the SSC based on the FPGA, and had a comparison between the ASK and the CCS. Finally, we have a study and debate about the function of anti-jamming and encryption.

\section{The related theory of the SSC}

The system of the SSC is the way which the spectrum of the message transmitted is spread by the special spread spectrum function, and then it is transmitted in the channel. And in the receiver, it uses the relevant method to compress it and the message is acquired. Also, the SSC is the system which transmits the same massage needs more band width than the various modulated way that we familiar, and the message band width is small than the message which has been spread. The message is not the important factor that defined the band width of the modulated message, the band width is defined by the spread spectrum function. And the common spread spectrum functions are the pseudo random functions. 
The basic theory of the SSC could be prescribed by the Shannon's formula about the volume of the channel:

The formula manifests that when the signal to noise ratio $(\mathrm{S} / \mathrm{N})$ of the transmitted system is declined, we can use the way of gain the system band width (W) to keep the volume of the channel steady. For the all special signal to noise ratio, we can make little signal error ratio by magnifying the transmitted channel. And the SSC is using the theory, which use the high rate spread spectrum code to spread the band width of the digital signal. And the density of the message power can be very low, and even the signal to noise ratio is very low and the message is submerged in the noise, we can still communicate formally.

\section{The design of the transmitter of the SSC}

\subsection{The functional block diagram of the transmitter of the SSC}

In the following, we use the pseudo random sequence as the spread spectrum function and construct the framework of transmitter, the figure 1 is the basic framework.

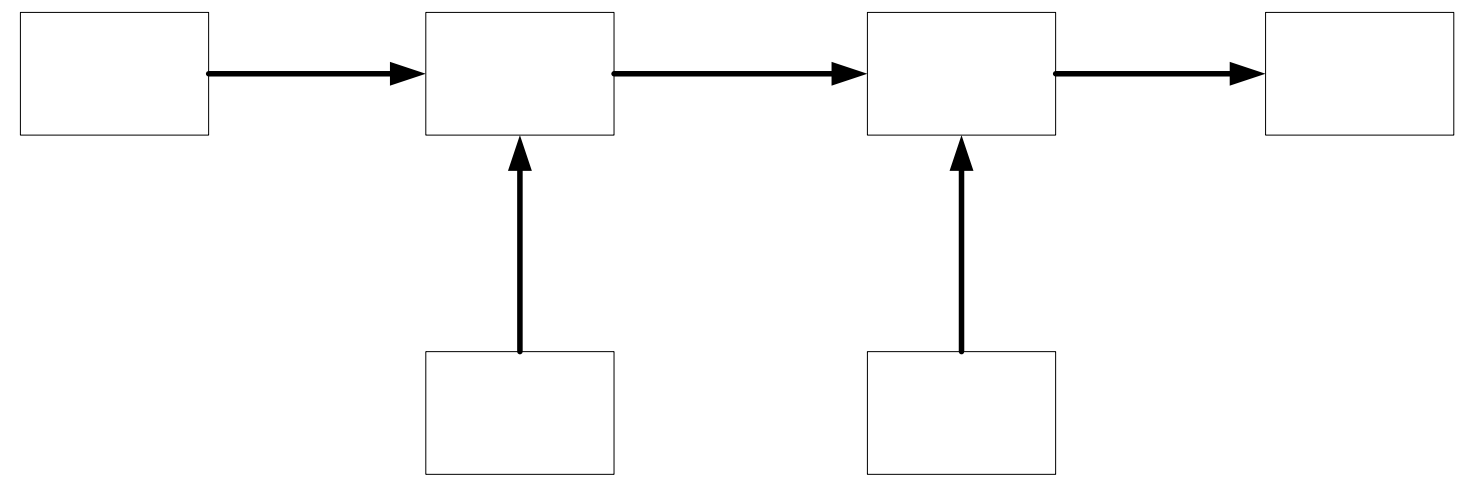

Fig.1 the functional block diagram of the transmitter of the SSC

In the figure 1, the signal a(t) output from the source is the message stream whose codes duration is Ta. The producer of the pseudo random code produces the high-speed pseudo random code $\mathrm{c}(\mathrm{t})$, the width of the element is Tc. We have a die 2 together about the message code $\mathrm{a}(\mathrm{t})$ and the pseudo random code $\mathrm{c}(\mathrm{t})$ and then we get a spread spectrum signal whose velocity is same with the pseudo random code. So, we get an extension about the spectrum of the signal, and then we can modulate the carrier and then we get an extended signal s(t).

\subsection{The design of the transmitter of the SSC}

In the transmitter of the SSC, the signal is produced by the source known, and it is our input module, and we select the MATLAB to produce it. The circuit which produces the PN pseudo noise) code is from the DSP Builder and then it is transferred to the VHDL (verilog hardware describe language), and then it will be used in the design. We can use the IP core in the Quartus as the oscillator module, and then we add some simple processing module and we can realize our design of the transmitter.

\subsubsection{The design of the producer of the PN sequence}

The SSC submerges the signal into the PN code, we choose the 4 order PN sequence as the spread spectrum code in order to the realization of the SSC and the result of the SSC. And because the band width of the spread spectrum code is more than 100 times of the signal, it means that the frequency which controls the PN code is 100 times more than the signal's, we can chose our frequency which controls the PN code according to the bit rate of the signal. 


\subsubsection{The realization of the DSP Builder for the circuit which produces the PN code}

At first, we should draw the framework of the 4 order $m$ sequence according to its primitive polynomial, and because the octal feedback coefficient is "23", the logical diagram is just like the figure 2 . Then we use the delay for shifting register and the exclusive-or gate for adder to realize the circuit, and the connected graph of the DSP Builder for the PN code is figure 3

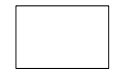

Fig.2 The logical diagram of the M sequence whose period is 15

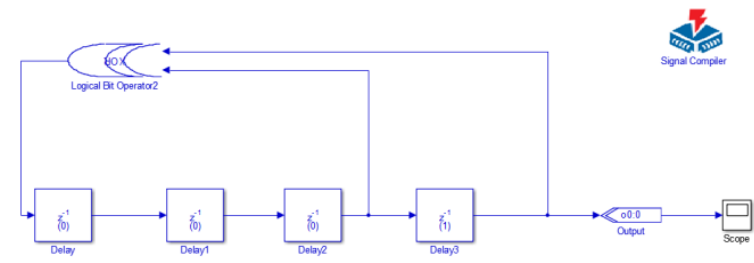

Fig.3 The connected graph of the DSP Builder for the PN code

\subsubsection{The realization of the electrical circuit for the PN code}

We use the module of signal compile in the DSP Builder to translate the connect graph into the project of the Quartus and run the project, the wave in the simulation is figure 4,it is same as expected, so we can use it to design the SSC.

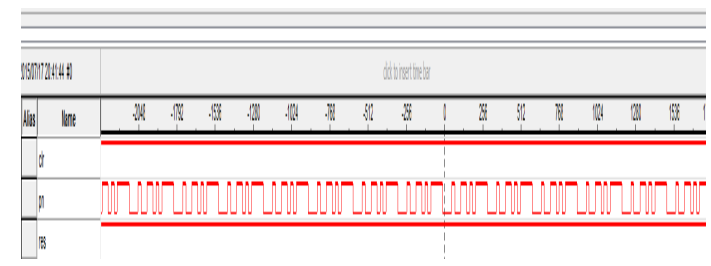

Fig. 4 The wave output from the project of the 4 orders PN code

\subsubsection{The design of the transmitters for the method of the SSC and ASK}

We design the transmitter of the SSC according to the functional block diagram in the figure 2,and then we design a ASK in the analog method of modulation. Then we put both of them into one project so that we can have a comparison between them, and finally, we program them into the FPGA.

\section{The analysis of the performance of the SSC}

\subsection{The comparison of the result of the two transmitters}

We have completed the design and the program of the transmitters of the SSC and the ASK, we can use the same source and the same frequency which control the system to have a simulation in the environment of the signaltap, the result is like the figure 5 . 


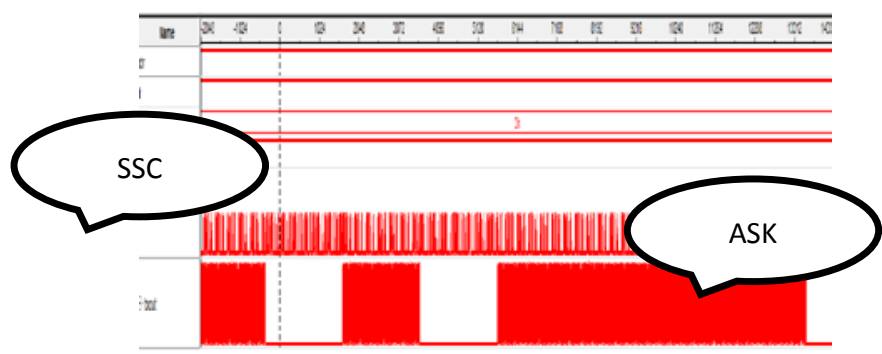

We can find that the wave output from the ASK is just the feedback of the message bit, it is the process of the carrier according to the message bit, while for the SSC, the output frequency bandwidth is very wide, the message is fully submerged into the noise, and output message in the all band is fairly homogeneous.

\subsection{The analysis of the SSC}

We can know from the wave above that the SSC uses the technology to submerge the message bit into the high-speed PN code, we can have a related detection in the receiver, and the useful signal will be a narrow-band, but for the interference, it will be extended because it is not correlated with the spread spectrum code. When the signal through a narrow-band filter, the power of the interference will be decreased, and also the signal to noise ratio will be increased, so it has a pretty strong function of anti-jamming.

Because the useful signal is broaden into the widen frequency band, the power of every unit will be very small, it also means that the useful message is in the noise, it is difficult to be detected and the useful parameters will also be difficult to be computed. In the another hand, if we want to intercept or capture the spread spectrum signal, we have to know the spread spectrum code of the SSC, and this will make more hinder, so it can achieve the purpose of the encryption.

\section{Conclusion}

The article has realized the miniaturization and the flexibility of the design of the transmitter, and also, it tests that the function of the anti-jamming and the encryption after the join of the spread spectrum code in the SSC by the comparison of the SSC and the ASK. But it doesn't have a comparison and analysis of the receiver when the noise is added, it just has a theoretical analysis on it. In the following research, I can make some analysis and comparison about the all procedure of the communication, and find more example and testify about it.

\section{References}

[1] Liang Zhaohui, Jiang Ting, Zhou Zheng. A New Synchronization scheme Using Perfect Punctured Binary Sequence Pairs in DS-UWB System [J].Journal of Electronics (China), Mar. 2006, Vol.23 No.2, 231-235.

[2] ZHANG Kai. Capture of PN sequence in the spread spectrum communication [J].Electronic Sci. and Tech., Dec. 2013,Vol.26 No.12, 34-36.

[3] SUN Jiapeng, ZHANG Jie, CHENG Hao, et al. Realizing the direct sequence spread spectrum communication with FPGA [J]. Journal of Inner Mongolia Agricultural University, Dec. 2008, Vol.29 No.4, 239-243.

[4] HAO Zhanghong, ZHAO Hongzhi, ZHAO Shihai, et al. A novel time-varying single tone jamming suppression method for DSSS system [J]. JOURNAL OF SIGNAL PROCESSING, Jul. 2014, Vol.30 No.7, 777-782.

[5] LI Zhaobin, JIANG Ting, ZOU Weixia, et al. Research on a new spread spectrum sequence pairs [J]. Journal of Electronics \& Information Technology, Apr. 2009, Vol. 31 No. 4, 889-892.

[6] GUO Lili, LI Beiming, DOU Zheng. The design of the spread spectrum communication system on the FPGA [M]. National Defense Industry Press, 2013.2, 2-3. 
[7] MA Jing, YIN Kuixi, KE Wei, et al. The Design and Implementation of a Spread Spectrum Communication Synchronous System Based on FPGA [J]. Microcomputer Information, 2006, Vol. 22 No.22, 175-177.

[8] HAO Zhanghong. Research on key technologies of time-varying interference suppression in direct sequence spread spectrum communication system [D]. Sichuan: University of Electronic Science and Technology of China, 2013, 6-7. 